\title{
Secondary infections in critically ill patients with COVID-19
}

\author{
Giacomo Grasselli, ${ }^{1}{ }^{*}$, Emanuele Cattaneo ${ }^{1,2}$ and Gaetano Florio ${ }^{1,2}$
}

\begin{abstract}
This article is one of ten reviews selected from the Annual Update in Intensive Care and Emergency Medicine 2021. Other selected articles can be found online at https://www.biomedcentral.com/collections/annualupdate2021. Further information about the Annual Update in Intensive Care and Emergency Medicine is available from https://link. springer.com/bookseries/8901.
\end{abstract}

\section{Introduction}

Since December 2019, when the first case of human transmission of the severe acute respiratory syndrome coronavirus 2 (SARS-CoV-2) was reported in Wuhan (China), more than a hundred million confirmed cases of coronavirus disease 2019 (COVID-19) have been described worldwide, and the pandemic declared on March 11, 2020 by the World Health Organization is still ongoing.

The clinical spectrum of SARS-CoV-2 infection ranges from asymptomatic disease to severe disease requiring hospitalization and admission to the intensive care unit (ICU) [1]. Recent multicenter studies showed that 5-32\% of hospitalized patients with COVID-19 needed ICU admission [2-5], mainly for acute respiratory distress syndrome (ARDS) requiring endotracheal intubation and invasive mechanical ventilation $[2-4,6,7]$. According to the available published data, the mortality of critically ill patients with COVID-19 ranges from 16 to $78 \%$ [3, 6-8].

For a number of reasons, patients with COVID-19 admitted to the ICU are at high risk of developing infectious complications during their ICU stay. First, they frequently develop multiple organ failure with need for vasopressors, renal replacement therapy (RRT) and, in

*Correspondence: giacomo.grasselli@unimi.it

${ }^{1}$ Department of Anesthesia, Intensive Care and Emergency, Fondazione IRCCS Ca' Granda Ospedale Maggiore Policlinico, Milan, Italy

Full list of author information is available at the end of the article some cases, extracorporeal membrane oxygenation support. The duration of mechanical ventilation and the ICU lengths of stay of these patients are therefore usually prolonged (up to 19 days for mechanical ventilation and up to 49 days for ICU length of stay [5, 9]). Second, COVID19 per se is associated with significant dysfunction of the patient's immune system. Multiple studies have shown the involvement of both innate and acquired immunity as a response to SARS-CoV-2 infection. Preliminary Chinese studies detected a reduction in both $\mathrm{CD} 4+\mathrm{T}$ and CD8+ T lymphocyte counts, an increase in neutrophils and a reduction in interferon gamma (IFN- $\gamma$ ) serum concentrations $[10,11]$. Further studies confirmed these findings and showed a cytokine pattern characterized by excess pro-inflammatory molecules (cytokine storm [12]), inhibition of natural killer cells (NK and NKT) and cytotoxic lymphocytes, and morphological and phenotypical alterations of monocytes [13-15]. Third, after the publication of the results of the RECOVERY trial [16], treatment with systemic corticosteroids has become standard of care in all patients requiring supplemental oxygen. In addition, a number of drugs aimed at blunting the immune system response to the viral infection (for example cytokine inhibitors [tocilizumab, anakinra, sarilumab] or complement inhibitors [eculizumab]) are frequently administered to these patients and several trials are ongoing to assess their efficacy. Finally, secondary bacterial and fungal infections as a complication of viral respiratory diseases have been described during previous 
pandemics (2002 severe acute respiratory syndrome [SARS] [17], 2009 swine influenza pandemic [18], and 2012 Middle East respiratory syndrome [MERS] [19]) and some studies have highlighted their role in increasing the severity of the viral pneumonia [18].

A recent review of the literature showed that the incidence of co-infections (i.e., infections detected at admission) in patients with COVID-19 is less than in previous pandemics [20]. Data on secondary infections (i.e., infections acquired during the course of ICU stay) are scarce. The aim of the present chapter is to summarize the available evidence on the epidemiology, risk factors, impact on outcome and principles of treatment of secondary infections in critically ill patients with COVID-19

\section{Epidemiology and risk factors Bacterial infections}

In patients with $\mathrm{H} 1 \mathrm{~N} 1$ influenza, the incidence of bacterial infections complicating the course of the viral pneumonia is $25-50 \%$ and they are associated with increased duration of mechanical ventilation, prolonged ICU stay and increased mortality [21]. For these reasons, early diagnosis and adequate management are mandatory in critically ill patients. As mentioned above, limited data are available on secondary bacterial infections in patients hospitalized for SARS-CoV-2 infection. A recent review reports a low incidence of bacterial or fungal infections in hospitalized COVID-19 patients, ranging from 6 to $15 \%$, but most of the cited studies were conducted in China and included patients admitted mainly to ordinary wards and not to the ICU; hence, these data cannot be extrapolated to the population of critically ill patients admitted to the ICU in western countries.

The most common bacterial complication of COVID19 is ventilator-associated lower respiratory tract infection (VA-LRTI), which includes ventilator-associated pneumonia (VAP) and ventilator-associated tracheobronchitis. The mechanism underlying bacterial coinfection in viral pneumonia is damage to the ciliated cells, which leads to impaired mucociliary clearance and increased adhesion of bacteria to mucins, resulting in enhanced bacterial colonization of the airways [22]. In addition to these mechanisms, other risk factors for bacterial secondary infections typical of ICU patients are the presence of ARDS and the prolonged duration of mechanical ventilation [23]. A recent multicenter European study described the cumulative incidence of VA-LRTI in patients with COVID-19 admitted to the ICU compared to patients with other viral and non-viral pneumonias. The overall incidence of VA-LRTI was $50 \%$, significantly higher than in the other two groups, despite the fact that patients with SARS-CoV-2 pneumonia had lower severity scores (Simplified Acute Physiology Score
[SAPS II] and Sequential Organ Failure Assessment [SOFA] score) at ICU admission and fewer comorbidities [24]. This finding has been confirmed by two other studies. The first evaluated the incidence of VAP in critically ill COVID-19 patients in the UK and showed that COVID-19 patients were significantly more likely to develop VAP than patients without COVID-19 [25]. The second study was a multicenter, observational trial conducted in several European countries and described the clinical characteristics of 4244 critically ill COVID-19 patients; the incidence of VAP in intubated patients was $58 \%$ [9]. As previously mentioned, prolonged duration of mechanical ventilation and a high incidence of ARDS, both typical of COVID-19, together with the administration of drugs affecting immune system function (in the multicenter European study $37.3 \%$ of the patients were treated with steroids) certainly contribute to this increased risk of secondary respiratory infections.

The most common bacteria involved in VA-LRTI in COVID-19 patients are Gram-negative bacilli, mainly Pseudomonas aeruginosa, Enterobacter spp. and Escherichia coli, followed by Gram-positive cocci, mainly Staphylococcus aureus [24]. Notably, some smaller reports describe different microorganism prevalence. For example, Sharifipour et al. [26] reported that among 19 ICU patients, secondary respiratory infections were caused by Acinetobacter baumannii in $90 \%$ of the cases and $S$. aureus in the remaining $10 \%$. However, the findings of these small, single-center case series are clearly influenced by the local epidemiology and are not representative of the general population of COVID-19 ICU patients.

The second most common secondary infections in critically ill COVID-19 patients are bloodstream infections (BSI). An Italian report estimated a cumulative risk of developing an episode of BSI of nearly $25 \%$ after 15 days of ICU stay and higher than $50 \%$ after 30 days. In multivariable analysis, anti-inflammatory treatment with tocilizumab or with methylprednisolone was independently associated with the development of BSI [27]. Buetti et al. [28] conducted a case control study comparing BSIs in 235 COVID-19 and 235 non-COVID 19 patients admitted to the ICU in France and described incidences of $14.9 \%$ and $3.4 \%$, respectively. In patients infected with SARS-CoV-2, BSIs occurred a median of 12 days after ICU admission. The most common microorganisms responsible for BSIs were coagulase-negative staphylococci $(36 \%)$. The authors also observed a significant increase in the risk of BSIs in COVID-19 patients treated with tocilizumab or anakinra [28] (Table 1).

\section{Fungal infections}

It is well known that viral pneumonia caused by influenza virus can facilitate the development of invasive 
Table 1 Characteristics and main findings of the studies describing secondary infections in patients with COVID-19

\begin{tabular}{|c|c|c|c|c|c|}
\hline Study [ref] & Sample size & Setting & $\begin{array}{l}\text { Incidence of } \\
\text { secondary } \\
\text { infections, \% }\end{array}$ & $\begin{array}{l}\text { Type and site of } \\
\text { infection (\%) }\end{array}$ & Microorganisms isolated (\%) \\
\hline Giacobbe et al. [27] & 78 & ICU & 40 & BSI (100) & Coag-neg staphylococci (24) E. faecalis (18) S. aureus (13) \\
\hline He et al. [46] & 918 & Hospital & 7 & $\begin{array}{l}\text { Pneumonia (32) } \\
\text { BSI (25) UTI (22) }\end{array}$ & $\begin{array}{l}\text { Coag-staphylococci (28) } \\
\text { A. baumannii (21) P. aeruginosa (14) }\end{array}$ \\
\hline Sharifipour et al. [26] & 19 & ICU & $100^{\mathrm{a}}$ & $\operatorname{VAP}(100)$ & A. baumannii (90) S. aureus (10) \\
\hline Fu et al. [47] & 36 & ICU & 14 & $\operatorname{VAP}(100)$ & S. mantophilia (40) \\
\hline Li et al. [48] & 1495 & Hospital & 7 & $\begin{array}{l}\text { Pneumonia (86) } \\
\text { BSI (34) UTI (8) }\end{array}$ & A. baumannii (36) K. pneumoniae (31) S. mantophilia (6) \\
\hline Rouzé et al. [24] & 568 & ICU & 51 & $\operatorname{VAP}(71)$ VAT (29) & P. aeruginosa (22) Enterobacter spp. (18) S. aureus (12) \\
\hline Buetti et al. [28] & 321 & ICU & 15 & BSI & $\begin{array}{l}\text { Coag-staphylococci (36) } \\
\text { Enterobacterales (13) P.aeruginosa (13) }\end{array}$ \\
\hline Dudoignon et al. [49] & 54 & $\mathrm{ICU}$ & 37 & $\operatorname{VAP}(75)$ & $\begin{array}{l}\text { P. aeruginosa (33) Enterobacteriaceae (33) } \\
\text { S. aureus (20) }\end{array}$ \\
\hline Ripa et al. [50] & 731 & Hospital & 9 & BSI (85) LRTI (32) & $\begin{array}{l}\text { Coag-staphylococci } 70 \% \text { of BSI } \\
\text { A. baumannii } 30 \% \text { of LRTI }\end{array}$ \\
\hline
\end{tabular}

BSI bloodstream infection, UTI urinary tract infection, VAP ventilator associated pneumonia, VAT ventilator associated tracheobronchitis, LRTI lower respiratory tract infection

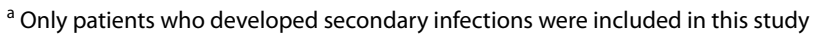

pulmonary aspergillosis, especially in patients presenting with ARDS, with a marked impact on the duration of hospitalization and mortality [29]. The limited data available in critically ill patients with COVID-19 seem to confirm the association between SARS-CoV-2 infection and development of invasive aspergillosis and some authors have suggested the existence of a clinical entity called COVID-19-associated pulmonary aspergillosis [30]. Risk factors for invasive pulmonary aspergillosis in COVID-19 patients are the direct lung damage due to the viral infection, use of corticosteroids, ARDS at presentation, treatment with broadspectrum antibiotics, and comorbidities [30]. The initial reports from China were very heterogeneous, describing an incidence of infection with Aspergillus ranging from $3 \%$ up to $23 \%$ among critically ill patients with COVID-19 [31, 32]. This variability could be due to the lack of precise definition criteria and of a standardized diagnostic algorithm for invasive pulmonary aspergillosis, possibly resulting in the underestimation of the real incidence of invasive pulmonary aspergillosis in some studies, while in others the misinterpretation of colonization may have led to an overestimation of the risk. European studies report a high rate (from 20\% to $35 \%$ ) of invasive pulmonary aspergillosis among critical patients with ARDS due to COVID-19, with a high mortality rate, ranging from 45 to $67 \%[33,34]$. The most common Aspergillus spp. responsible for invasive pulmonary aspergillosis in these patients seems to be Aspergillus fumigatus (isolated in $90 \%$ of the cultures), followed by Aspergillus flavus [30].

\section{Diagnosis of secondary infections}

\section{Bacterial infections}

As previously mentioned, the most common secondary infections in critically ill patients with COVID-19 are VAP and BSIs. The diagnosis is made when the patient shows clinical symptoms and signs of infection and a new pathogen is detected in a biological specimen.

VAP is defined as the association of persistent pulmonary infiltrates on radiological imaging and positive microbiological cultures from a lower respiratory tract specimen with clinical suspicion of new onset pneumonia in a patient that has received at least $48 \mathrm{~h}$ of invasive mechanical ventilation [35-37]. Scores, such as the Clinical Pulmonary Infection Score (CPIS) [38] (based on six variables: temperature, blood leukocytes, aspect of tracheal secretions, oxygenation, radiographic infiltrates, and Gram stain on tracheal aspirates), have been developed to help clinicians diagnose VAP, but the most recent guidelines [36, 39] highlight the role of clinical signs of infection (i.e., new onset of fever, purulent secretion from the air-way, leukocytosis or leukopenia, worsening of blood oxygenation, increased need for inotropic and vasoactive agents) rather than the use of a score. Imaging techniques, such as chest X-ray [37], chest computed tomography, and, more recently, lung ultrasound [40, 41], tailored to detect new pulmonary infiltrates, and markers of inflammation (e.g., C-reactive protein, procalcitonin) can support the clinical diagnosis.

Adequate and specific antibiotic therapy, however, requires a microbiological diagnosis based on culture examinations and tests (e.g., Gram stain, biomarkers, 
rapid diagnostic assay, polymerase chain reaction [PCR]) to enable identification of the involved bacteria. Samples can be obtained from the distal airway in a more invasive way using bronchoscopy (i.e., bronchoalveolar lavage [BAL], protected specimen brush [PSB]), in a 'less-invasive' way (i.e., blind mini-BAL, blind PSB) or from the proximal airway (endotracheal aspirate); a recent metaanalysis [42] comparing cultures from proximal and distal airways showed no differences in patient outcome, but it should be remembered that sampling from the distal airway may be associated with an increased risk for the patient (i.e., hypoxemia, bleeding). Furthermore, invasive procedures are associated with potential exposure to aerosolized viral particles, which represents a risk for healthcare personnel.

BSI in critically ill patients is defined as the onset of signs and symptoms of infection within $24 \mathrm{~h}$ of a positive blood culture. Blood cultures and identification of specific bacteria represent the gold standard for the diagnosis, but a single positive culture is not suggestive of infection when a typical human skin contaminant is involved; in this case, the diagnosis requires at least two positive blood cultures for the microorganism within $48 \mathrm{~h}$.

\section{Fungal infections}

COVID-19-associated pulmonary aspergillosis should be suspected in all patients with COVID-19 who present with refractory fever lasting more than 3 days after an initial 48-h period of defervescence (following appropriate antibiotic therapy), worsening of gas exchange, onset of hemoptysis, or new pleural rubs [29]. A complete and accurate algorithm for diagnosing COVID-19-associated pulmonary aspergillosis is still lacking but it would be useful to search for Aspergillus spp. in respiratory samples (e.g., bronchoalveolar lavage, tracheal aspirate) and to use serologic biomarkers such as galactomannan on respiratory samples and serum. Other tests that may help in diagnosing COVID-19-associated pulmonary aspergillosis are aspergillus PCR and serum $(1 \rightarrow 3)-\beta$-D-glucan.

\section{Principles of treatment \\ Bacterial infections}

The initial Surviving Sepsis Campaign guidelines for the management of critically ill patients with COVID-19 suggested an empiric antibacterial agent in all mechanically ventilated patients [43]. However, subsequent data have shown that, at ICU admission, patients infected with SARS-CoV-2 seldom have concomitant bacterial infection. For this reason, and because of the high incidence of infectious complications caused by multidrug-resistant (MDR) germs, most experts agree that prophylactic administration of an empiric antibiotic therapy in the absence of clear signs of a co-infection or of a secondary infection should be discouraged. Indeed, it has been demonstrated that inappropriate initial antimicrobial treatment is associated with increased mortality in VAP and with increased bacterial resistance [44]. In addition, in critically ill patients, different doses from those usually recommended may be used, either because normal doses may not achieve effective drug concentrations at the target site or because they can be associated with adverse reactions due to toxic concentrations. For these reasons, therapeutic drug monitoring of plasma trough levels is recommended.

Available guidelines $[35,36]$ recommend that empirical therapy should be started as soon as VAP is clinically suspected. The empirical therapy should be modified based on the results of the culture tests. The choice of the empirical treatment is based mainly on the patient's risk factors for MDR pathogens, and on the local pattern of antimicrobial susceptibility. Among the risk factors for MDR is ARDS prior to VAP and hospital stay $>5$ days, both very likely to be present in COVID-19 patients. In this case, the empirical treatment of choice should be a broad-spectrum anti-pseudomonas $\beta$-lactam plus a non- $\beta$-lactam antipseudomonal agent (e.g., piperacillin-tazobactam plus amikacin). When choosing the antibiotic, it is important to consider the local pattern of susceptibility, and the results of microbiological surveillance for patient colonization. Empiric coverage of methicillin-resistant $S$. aureus (MRSA) should be considered in units where the incidence of VAP is higher than 20\% [35]. Once culture and susceptibility results are obtained, the main goal should be to remove unnecessary antibiotics (especially anti-MRSA and carbapenems) and use a narrow spectrum agent if possible.

\section{Fungal infections}

Patients with invasive aspergillosis often have many comorbidities that, together with the underlying disease, can affect the pharmacokinetics of antifungal medications. As reported earlier for antibacterial agents, even for antimycotics the risk of not reaching the target concentration at the infection site or of toxicity exists, especially in critically ill patients, thus therapeutic drug monitoring is recommended. Given the high mortality rate of patients with critical COVID-19 and concomitant invasive pulmonary aspergillosis, treatment should be started as soon as the diagnosis of invasive pulmonary aspergillosis is made.

Voriconazole is recommended as first line treatment in invasive pulmonary aspergillosis, with a target plasma trough concentration of 2-6 mg/l. Repeated monitoring is indicated until steady-state level is confirmed or if there is a change in the patient's clinical condition or suspected toxicity. In patients with liver dysfunction or when voriconazole cannot be administered, liposomal amphotericin 
$\mathrm{B}$ is appropriate. In patients who do not respond or do not tolerate initial therapy an echino-candin alone or in combination with voriconazole is indicated [45].

\section{Conclusion}

Secondary infections, frequently caused by MDR germs, are common in critically ill patients with COVID-19 admitted to the ICU, as a result of a number of favoring conditions. Early and accurate diagnosis and institution of adequate antimicrobial treatment are essential to improve patient outcome. Preliminary published data indicate that secondary infections are associated with increased duration of mechanical ventilation and of ICU stay, and that they may have an impact on patient survival. However, data from large, well-designed studies are needed to confirm these findings and to improve our knowledge of the epidemiology and treatment of infections complicating the clinical course of COVID-19.

\section{Acknowledgements}

Not applicable.

\section{Authors' contributions}

All authors contributed to the study conception and design. Literature search and data analysis were performed by EC and GF. The first draft of the manuscript was written by GG and EC and GF commented on previous versions of the manuscript and reviewed the manuscript for important intellectual content. All authors read and approved the final manuscript.

\section{Funding}

This study was funded by institutional funds (Ricerca Corrente 2021) of the Fondazione IRCCS Ca' Granda Ospedale Maggiore Policlinico, Milan, Italy and by a Grant Ricerca Finalizzata, Italian Ministry of Health (Project PB-0154 PROGETTO COVID-2020-12371675 "COVID19: epidemiological, clinical, genetic and social determinants of infection and disease progression"). Publication costs were funded by Grant Ricerca Finalizzata, Italian Ministry of Health (Project PB-0154).

\section{Availability of data and materials}

All data generated or analyzed during this study are included in this published article (and its supplementary information files).

\section{Declarations}

Ethics approval and consent to participate Not applicable.

\section{Consent for publication}

Not applicable.

\section{Competing interests}

Dr. Grasselli reports personal fees from Maquet, personal fees from Biotest, personal fees from Pfizer, personal fees from Draeger, personal fees from Fisher\&Paykel, personal fees from MSD.

\section{Author details}

${ }^{1}$ Department of Anesthesia, Intensive Care and Emergency, Fondazione IRCCS Ca'Granda Ospedale Maggiore Policlinico, Milan, Italy. ${ }^{2}$ Department of Pathophysiology and Transplantation, University of Milan, Milan, Italy.

Published online: 31 August 2021

\section{References}

1. Grasselli G, Pesenti A, Cecconi M. Critical care utilization for the COVID-19 outbreak in Lombardy, Italy. JAMA. 2020;323:1545-6.

2. Guan W, Ni Z, Hu Y, Liang W, Ou C, He J, et al. Clinical characteristics of coronavirus disease 2019 in China. N Engl J Med. 2020;382:1708-20.

3. Huang C, Wang Y, Li X, Ren L, Zhao J, Hu Y, et al. Clinical features of patients infected with 2019 novel coronavirus in Wuhan, China. Lancet. 2020;395:497-506.

4. Grasselli G, Zangrillo A, Zanella A, Antonelli M, Cabrini L, Castelli A, et al. Baseline characteristics and outcomes of 1591 patients infected with SARS-CoV-2 admitted to ICUs of the Lombardy region, Italy. . JAMA. 2020;323:1574-81.

5. Grasselli G, Greco M, Zanella A, Albano G, Antonelli M, Bellani G, et al. Risk factors associated with mortality among patients with COVID-19 in intensive care units in Lombardy, Italy. JAMA Intern Med. 2020;180:1345-55.

6. Arentz M, Yim E, Klaff L, Lokhandwala S, Riedo FX, Chong M, et al. Characteristics and outcomes of 21 critically ill patients with COVID-19 in Washington State. JAMA. 2020;323:1612-4.

7. Wang D, Hu B, Hu C, Zhu F, Liu X, Zhang J, et al. Clinical characteristics of 138 hospitalized patients with 2019 novel coronavirus-infected pneumonia in Wuhan, China. JAMA. 2020;323:1061-9.

8. Zhou F, Yu T, Du R, Fan G, Liu Y, Liu Z, et al. Clinical course and risk factors for mortality of adult inpatients with COVID-19 in Wuhan, China: a retrospective cohort study. Lancet. 2020;395:1054-62.

9. Schmidt M, Hajage D, Demoule A, Pham T, Combes A, Dres M, et al. Clinical characteristics and day-90 outcomes of 4244 critically ill adults with COVID-19: a prospective cohort study. Intensive Care Med. 2021;47:60-73.

10. Qin C, Zhou L, Hu Z, Zhang S, Yang S, Tao Y, et al. Dysregulation of immune response in patients with coronavirus 2019 (COVID-19) in Wuhan, China. Clin Infect Dis. 2020;71:762-8.

11. Liu J, Liu Y, Xiang P, Pu L, Xiong H, Li C, et al. Neutrophil-to-lymphocyte ratio predicts critical illness patients with 2019 coronavirus disease in the early stage. J Transl Med. 2020;18:206.

12. Mehta P, McAuley DF, Brown M, Sanchez E, Tattersall RS, Manson JJ, et al. COVID-19: consider cytokine storm syndromes and immunosuppression. Lancet. 2020;395:1033-4.

13. Zhang JJ, Dong X, Cao YY, Yuan YD, Yang YB, Yan YQ, et al. Clinical characteristics of 140 patients infected with SARS-CoV-2 in Wuhan, China. Allergy. 2020;75:1730-41.

14. Xiong Y, Liu Y, Cao L, Wang D, Guo M, Jiang A, et al. Transcriptomic characteristics of bronchoalveolar lavage fluid and peripheral blood mononuclear cells in COVID-19 patients. Emerg Microbes Infect. 2020;9:761-70.

15. Zheng M, Gao Y, Wang G, Song G, Liu S, Sun D, et al. Functional exhaustion of antiviral lymphocytes in COVID-19 patients. Cell Mol Immunol. 2020;17:533-5.

16. The RECOVERY Collaborative Group. Dexamethasone in hospitalized patients with COVID-19. N Engl J Med. 2021;384:693-704.

17. Wilder-Smith A, Green JA, Paton NI. Hospitalized patients with bacterial infections: a potential focus of SARS transmission during an outbreak. Epidemiol Infect. 2004;132:407-8.

18. Morris DE, Cleary DW, Clarke SC. Secondary bacterial infections associated with influenza pandemics. Front Microbiol. 2017:8:1041.

19. Memish ZA, Perlman S, Van Kerkhove MD, Zumla A. Middle East respiratory syndrome. Lancet. 2020;395:1063-77.

20. Fattorini L, Creti R, Palma C, Pantosti A. Unit of Antibiotic Resistance and Special Pathogens, Unit of Antibiotic Resistance and Special Pathogens of the Department of Infectious Diseases, Istituto Superiore di Sanità R. Bacterial coinfections in COVID-19: an underestimated adversary. Ann Ist Super Sanita. 2020;56:359-64.

21. Papazian L, Klompas M, Luyt CE. Ventilator-associated pneumonia in adults: a narrative review. Intensive Care Med. 2020;46:888-906.

22. Wilson R, Dowling RB, Jackson AD. The biology of bacterial colonization and invasion of the respiratory mucosa. Eur Respir J. 1996;9:1523-30.

23. Forel J-M, Voillet F, Pulina D, Gacouin A, Perrin G, Barrau K, et al. Ventilatorassociated pneumonia and ICU mortality in severe ARDS patients ventilated according to a lung-protective strategy. Crit Care. 2012;16:R65.

24. Rouzé A, Martin-Loeches I, Povoa P, Makris D, Artigas A, Bouchereau M, et al. Relationship between SARS-CoV-2 infection and the incidence of ventilator-associated lower respiratory tract infections: a European multicenter cohort study. Intensive Care Med. 2021;47:188-98. 
25. Maes M, Higginson E, Pereira-Dias J, Curran MD, Parmar S, Khokhar F, et al. Ventilator-associated pneumonia in critically ill patients with COVID-19. Crit Care. 2021;25:25.

26. Sharifipour E, Shams S, Esmkhani M, Khodadadi J, Fotouhi-Ardakani R, Koohpaei A, et al. Evaluation of bacterial co-infections of the respiratory tract in COVID-19 patients admitted to ICU. BMC Infect Dis. 2020;20:646.

27. Giacobbe DR, Battaglini D, Ball L, Brunetti I, Bruzzone B, Codda G, et al. Bloodstream infections in critically ill patients with COVID-19. Eur J Clin Invest. 2020;50:e13319.

28. Buetti N, Ruckly S, de Montmollin E, Reignier J, Terzi N, Cohen Y, et al. COVID-19 increased the risk of ICU-acquired bloodstream infections: a case-cohort study from the multicentric OUTCOMEREA network. Intensive Care Med. 2021:47:180-7.

29. Schauwvlieghe AFAD, Rijnders BJA, Philips N, Verwijs R, Vanderbeke L, Van Tienen C, et al. Invasive aspergillosis in patients admitted to the intensive care unit with severe influenza: a retrospective cohort study. Lancet Respir Med. 2018;6:782-92.

30. Arastehfar A, Carvalho A, van de Veerdonk FL, Jenks JD, Koehler P, Krause $\mathrm{R}$, et al. COVID-19 associated pulmonary aspergillosis (CAPA)—from immunology to treatment. J Fungi. 2020;6:91.

31. Zhang G, Hu C, Luo L, Fang F, Chen Y, Li J, et al. Clinical features and shortterm outcomes of 221 patients with COVID-19 in Wuhan, China. J Clin Virol. 2020;127:104364.

32. Zhu $X, G e Y$, Wu T, Zhao K, Chen Y, Wu B, et al. Co-infection with respiratory pathogens among COVID-2019 cases. Virus Res. 2020;285:198005.

33. Alanio A, Dellière S, Fodil S, Bretagne S, Mégarbane B. Prevalence of putative invasive pulmonary aspergillosis in critically ill patients with COVID-19. Lancet Respir Med. 2020;8:e48-9.

34. van Arkel ALE, Rijpstra TA, Belderbos HNA, van Wijngaarden P, Verweij PE, Bentvelsen RG. COVID-19-associated pulmonary aspergillosis. Am J Respir Crit Care Med. 2020;202:132-5.

35. Torres A, Niederman MS, Chastre J, Ewig S, Fernandez-Vandellos P, Hanberger $\mathrm{H}$, et al. International ERS/ESICM/ESCMID/ALAT guidelines for the management of hospital-acquired pneumonia and ventilator-associated pneumonia: Guidelines for the management of hospital-acquired pneumonia (HAP)/ventilator-associated pneumonia (VAP) of the European Respiratory Society (ERS), European Society of Intensive Care Medicine (ESICM), European Society of Clinical Microbiology and Infectious Diseases (ESCMID) and Asociación Latinoamericana del Tórax (ALAT). Eur Respir J. 2017;50:1700582.

36. Kalil AC, Metersky ML, Klompas M, Muscedere J, Sweeney DA, Palmer $L B$, et al. Management of adults with hospital-acquired and ventilatorassociated pneumonia: 2016 Clinical Practice Guidelines by the Infectious Diseases Society of America and the American Thoracic Society. Clin Infect Dis. 2016;63:e61-111.

37. Chastre J, Luyt C-E. Does this patient have VAP? Intensive Care Med. 2016:42(7):1159-63.

38. Pugin J, Auckenthaler R, Mili N, Janssens JP, Lew PD, Suter PM. Diagnosis of ventilator-associated pneumonia by bacteriologic analysis of bronchoscopic and nonbronchoscopic "blind" bronchoalveolar lavage fluid. Am Rev Respir Dis. 1991;143:1121-9.

39. Leone M, Bouadma L, Bouhemad B, Brissaud O, Dauger S, Gibot S, et al. Brief summary of French guidelines for the prevention, diagnosis and treatment of hospital-acquired pneumonia in ICU. Ann Intensive Care. 2018;8:104.

40. Bouhemad B, Dransart-Rayé O, Mojoli F, Mongodi S. Lung ultrasound for diagnosis and monitoring of ventilator-associated pneumonia. Ann Transl Med. 2018;6:418

41. Mongodi S, Via G, Girard M, Rouquette I, Misset B, Braschi A, et al. Lung ultrasound for early diagnosis of ventilator-associated pneumonia. Chest 2016;149:969-80

42. Berton DC, Kalil AC, Teixeira PJZ. Quantitative versus qualitative cultures of respiratory secretions for clinical outcomes in patients with ventilatorassociated pneumonia. Cochrane Database Syst Rev. 2014;30:CD006482.

43. Alhazzani W, Møller MH, Arabi YM, Loeb M, Gong MN, Fan E, et al. Surviving Sepsis Campaign: guidelines on the management of critically ill adults with Coronavirus Disease 2019 (COVID-19). Intensive Care Med. 2020;46:854-87.

44. Iregui M, Ward S, Sherman G, Fraser VJ, Kollef MH. Clinical importance of delays in the initiation of appropriate antibiotic treatment for ventilatorassociated pneumonia. Chest. 2002;122:262-8.

45. Ullmann AJ, Aguado JM, Arikan-Akdagli S, Denning DW, Groll AH, Lagrou $K$, et al. Diagnosis and management of Aspergillus diseases: executive summary of the 2017 ESCMID-ECMM- ERS guideline. Clin Microbiol Infect. 2018;24(Suppl 1):e1-38.

46. He Y, Li W, Wang Z, Chen H, Tian LLD. Nosocomial infection among patients with COVID-19: A retrospective data analysis of 918 cases from a single center in Wuhan, China. . Infect Control Hosp Epidemiol. 2020:41:982-3.

47. Fu Y, Yang $Q, X u M$, et al. Secondary bacterial infections in critical ill patients with coronavirus disease 2019. Open Forum Infect Dis. 2020;7:ofaa220

48. Li J, Wang J, Yang Y, Cai P, Cao J, Cai XZY. Etiology and antimicrobial resistance of secondary bacterial infections in patients hospitalized with COVID-19 in Wuhan, China: a retrospective analysis. Antimicrob Resist Infect Control. 2020;9:153.

49. Dudoignon E, Caméléna F, Deniau B, et al. Bacterial pneumonia in COVID19 critically ill patients: a case series. Clin Infect Dis. 2021;72:905-6.

50. Ripa M, Galli L, Poli A, Oltolini C, Spagnuolo V, Mastrangelo A, et al. Secondary infections in patients hospitalized with COVID-19: incidence and predictive factors. Clin Microbiol Infect. 2021;27:451-7.

\section{Publisher's Note}

Springer Nature remains neutral with regard to jurisdictional claims in published maps and institutional affiliations. 